\title{
Investigating the Performance of an Electrothermally Actuated Resonant Microgyroscope with Decoupled Structure
}

\author{
S. A. Afzal and M. Hamedi
}

\begin{abstract}
This paper introduces an electrothermally actuated resonant microgyroscope and shows that how a thermal actuator can have a proper performance in the drive mode. In the proposed microgyroscope a v-shaped electro thermal actuator is utilized to drive the proof mass in the drive mode whereas parallel plate electrodes are used for sensing the rotation induced Coriolis force in the sense mode. An intermediate mass and decoupling beams decouples the drive and sense mode that leads to decreasing mechanical crosstalk. Higher sensitivity is achieved by designing the drive and sense mode resonant frequencies being close to each other. The analytical analysis was done based on the motion equations after which the resonant frequency of drive and sense mode were calculated. The modal analysis, which was performed using COMSOL, predicted the drive and sense resonant frequency at $2001 \mathrm{~Hz}$ and $1998 \mathrm{~Hz}$ respectively. The results of both methods were in satisfactory agreement. In addition, the function of the gyroscope was simulated while applying a sinusoidal voltage. The frequency response of the drive mode was measured both using analytical equations and numerical simulations which showed a good agreement. The amplitude of the displacement in the drive mode at resonant frequency presented that an electrothermal actuator can be suitably excite proof mass. The advantages of an electrothermal actuator over an electrostatic actuator in the drive mode are; low damping factor; low excitation voltage and electrical circuits of lower complexity.
\end{abstract}

Index Terms-Microgyroscope, electrothermal actuator, resonant frequency, frequency response.

\section{INTRODUCTION}

Microgyroscopes are one the most important microelectromechanical sensors whose features such as reduction in weight, size, and energy consumption make them widespread [1], [2]. These sensors are applicable in navigation systems, ride stabilization, rollover detection, medical instruments and other areas [3], [4]. Typically the overall dynamical system of a resonant micromachined vibratory gyroscope is a 2-DoF mass-spring-damper system [5]. In this type of sensors, an external actuator excites the drive mode oscillator into resonance in the drive direction. When a gyroscope is under an angular rotation, a sinusoidal Coriolis force at the driving frequency which is orthogonal to the drive direction and the input angular rate axis is induced and displaces the proof mass in the sense direction. By measuring the vibration amplitude in the sense direction, it is possible to determine the angular rate of the system [6].

Manuscript received February 25, 2017, revised June 3, 2017.

The authors are with University of Tehran, Tehran, 14399-57131, Iran (e-mail: s_aliafzal@alumni.ut.ac.ir,mhamedi@ut.ac.ir).
Since the previous decade, various resonant microgyroscopes with different structures and characteristics have been designed and fabricated. However, most of the new structures and characteristics are implemented to decouple the sense and the drive modes which leads to minimize the mechanical crosstalk [7], [10]. In recent years, electrostatic comb drive actuators (ECDAs) are widely used in many gyroscopes as the driving mechanism [10], [12]. Although, this kind of actuator shows proper accuracy and performance, a complex electrical circuit is required to drive it. Moreover, the atmospheric pressure around ECDA causes proportionally high damping effect. Considering the points mentioned above, it is essential that we apply some other types of actuator for driving the proof mass of the gyroscope in the drive mode. While the response time of the electrothermal actuator is proportionally low, it seems that the application of this kind of actuators is impossible being used to excite the drive mode oscillator. Since the time when the first microgyroscope was fabricated in Draper laboratory, the application of electrothermal actuator as actuation mechanism has been rarely used. In 2011, one of the first samples of micgyroscopes with this kind of actuator was designed and fabricated [13]. The results in the study showed that, it is possible to use electrothermal actuator as drive mechanism. However, there is no explanation for how an electrothermal actuator can operate at relatively high frequency, theoretically. The response time of sensors and actuators proportional to their size; the smaller a sensor or actuator is made, the faster it will respond [14].

In this paper, a microgyroscope which uses the $\mathrm{V}$-shaped thermal actuator to excite the proof mass oscillator is designed. Further, the performance of the microgyroscope was studied which showed that an electrothermal actuator could be applicable. Section II presents the design of suspension system. In Section III, the resonant frequency in both the drive and sense modes using numerical and analytical methods are determined. Section IV points to the heat transfer phenomenon in microelectrothermal actuators. In Section V, the performance of electrothermal actuator is simulated. Section VI explains the damping caused by atmospheric pressure. In Section VII, the frequency response of the drive mode is calculated using both numerical and analytical methods. Finally, Section VIII concludes the aforementioned arguments.

\section{GYROSCOPE DESIGN}

The design of micromachined vibratory gyroscopes is very complex since several parameters should be considered 
simultaneously. To increase the performance of the device, the resonant frequency of sense and drive mode should be close enough, commonly called match mode. Moreover, the vibration of the drive and sense mode should be decoupled to reduce the mechanical crosstalk. Coriolis force enhancement and Brownian noise reduction requires the mass to be larger. However this approach is hard to implement because of the limitations on the technology of MEMS process [10].

Fig. 1 shows the configuration of the proposed nickel-based microgyroscope. An electrothermal actuator is used, whereas a parallel plate electrode is used for sensing the rotation. The $\mathrm{V}$-shaped electrothermal actuator consists of two hot arms at a small angle with respect to each other [14]. Metals are much better for this kind of actuators due to provide relatively large displacement in low working temperature [13]. A nickel based actuator is used in this study which results almost $60 \%$ more displacement in comparison to a silicon based one [15].

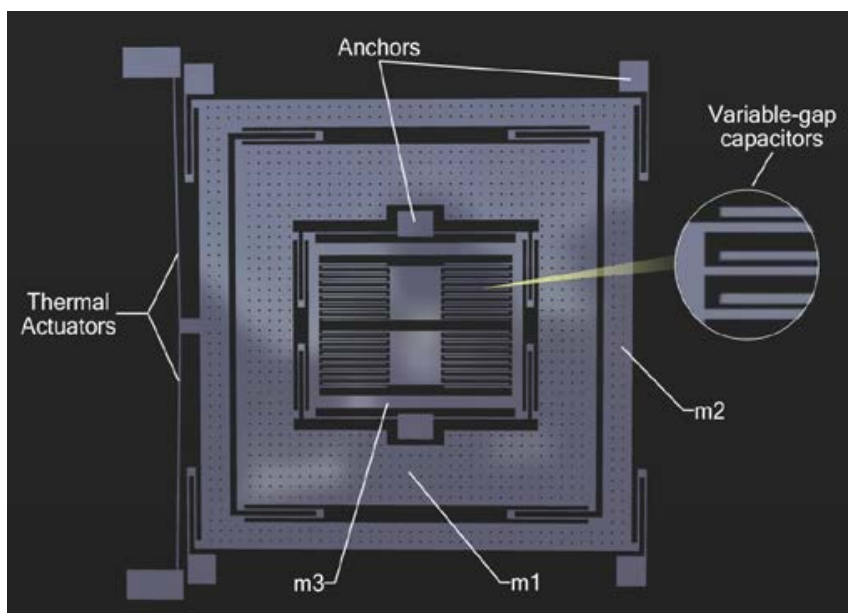

Fig. 1. Simplified schematic of the designed microgyroscopes.

The other advantages of the application of this kind of actuator are simple use; not requiring complex circuits; providing considerable displacement; and the low damping coefficient. As it is shown in Fig. 1 the proposed microgyroscope composed of 3 masses. $m_{1}, m_{2}$, and $m_{3}$ which are coupled though a frame, are named intermediate mass, drive mass, and sense mass, respectively. Drive and sense mass has one degree of freedom while intermediate mass has two DoFs. Applying $\mathrm{m}_{1}$ as an intermediate mass decouples the drive and the sense mode oscillations to minimize mechanical crosstalk.

Actuator excites the $\mathrm{m}_{1}$ and $\mathrm{m}_{2}$ along drive direction in resonance. If gyroscope is forced to rotate along $\mathrm{z}$ direction, $\mathrm{m}_{3}$ will displace in sense direction due to coriolis force.

\section{A. Suspension Design}

In Fig. 2 the complete configuration of the suspension system is shown. The system consists of 12 double folded flexures and 4 fixed-guided beams. The suspension which connects $m_{2}$ to the substrate via anchor consists of four double folded flexures. Every double folded flexure can be modeled as two fixed-guided beams. The beams deformed orthogonally to the axis of the beam. The equivalent stiffness of the beams with length of $\mathrm{L}_{11 \mathrm{x}}$ and $\mathrm{L}_{12 \mathrm{x}}$ in drive direction can be calculated using the following equations [6].

$$
\begin{aligned}
& k_{1 x}=4\left(\frac{k_{11 x}+k_{12 x}}{k_{11 x} \cdot k_{12 x}}\right) \\
& k_{11 x}=\frac{1}{2} \frac{3 E I}{\left(L_{11 x} / 2\right)^{3}}=\frac{E t w^{3}}{L_{11 x}{ }^{3}} \\
& k_{12 x}=\frac{1}{2} \frac{3 E I}{\left(L_{12 x} / 2\right)^{3}}=\frac{E t w^{3}}{L_{12 x}{ }^{3}}
\end{aligned}
$$

where $w, t$ and $I=t w^{3} / 12$ are width, thickness and the second moments of inertia of the beam respectively. The intermediate mass is connected to drive mass via 4 double folded flexures which enable it to displace in drive direction. The equivalent stiffness of the beams in drive direction whose lengths are $L_{2 x}$ is calculated using the following equation [6].

$$
k_{2 x}=\frac{4}{2}\left(\frac{1}{2} \frac{3 E I}{\left(L_{2 x} / 2\right)^{3}}\right)=\frac{2 E t w^{3}}{L_{2 x}^{3}}
$$

If we neglect the small angle change, we can model the v-shaped actuator as a fixed-fixed beam. The equivalent stiffness of the actuators whose lengths are $L_{C}$ in drive direction is calculated by Eq. 5 [6].

$$
k_{c}=2\left(\frac{192 E I}{L_{C}{ }^{3}}\right)=2\left(\frac{16 E t w_{C}{ }^{3}}{L_{C}{ }^{3}}\right)
$$

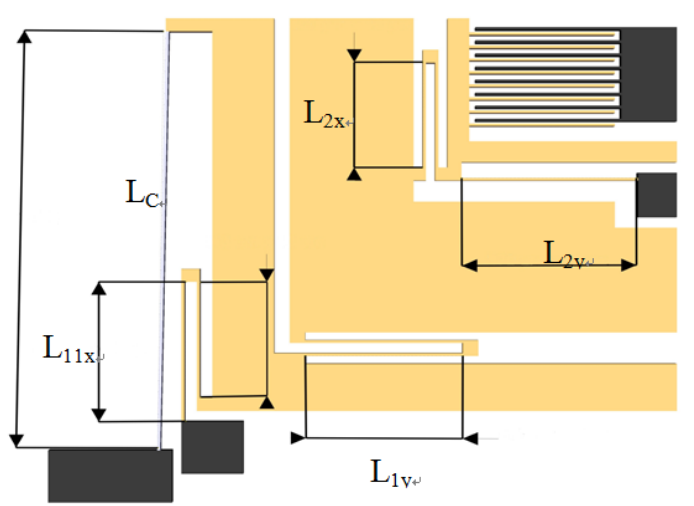

Fig. 2. Microgyroscope suspension system layout.

The intermediate mass is connected to sense mass via 4 double folded flexures which enable it to displace in sense direction. The equivalent stiffness in sense direction whose lengths are $L_{2 y}$ is calculated using the following equation [6].

$$
k_{1 y}=\frac{4}{2}\left(\frac{1}{2} \frac{3 E I}{\left(L_{1 y} / 2\right)^{3}}\right)=\frac{2 E t w^{3}}{L_{1 y}{ }^{3}}
$$

The suspension which connects $m_{3}$ to the substrate via anchor consists of four fixed-guided beams which can displace in sense direction. The equivalent stiffness for the beams whose lengths are $L_{2 y}$ is described using Eq. 7

$$
k_{2 y}=4\left(\frac{1}{2} \frac{3 E I}{\left(L_{2 y} / 2\right)^{3}}\right)=\frac{4 E t w^{3}}{L_{2 y}{ }^{3}}
$$

The stiffness of the system in drive and sense direction are 
yielded by summing Eq1-Eq5 and Eq6-Eq7 respectively.

\section{ANALYSIS}

\section{A. Analytical Analysis for Resonant Frequency Determination}

The resonant frequency in drive and sense mode is calculated using Eq. 8 and 9.

$$
\begin{aligned}
& w_{x}=\frac{1}{2 \pi} \sqrt{\frac{k_{x}}{m_{x}}}=\frac{1}{2 \pi} \sqrt{\frac{k_{1 x}+k_{2 x}+k_{C}}{m_{1}+m_{2}}} \\
& w_{y}=\frac{1}{2 \pi} \sqrt{\frac{k_{y}}{m_{y}}}=\frac{1}{2 \pi} \sqrt{\frac{k_{1 y}+k_{2 y}}{m_{1}+m_{3}}}
\end{aligned}
$$

Different dimensions of suspension systems is given in Table I.

TABle I.: THE VALUE FOR THE DIFFERENT DIMENSIONS

\begin{tabular}{|c|c|c|c|}
\hline Parameter & Value & Parameter & Value \\
\hline$L_{11 x}$ & $533 \mu \mathrm{m}$ & $L_{1 y}$ & $509 \mu \mathrm{m}$ \\
\hline$L_{12 x}$ & $443 \mu \mathrm{m}$ & $L_{2 y}$ & $570 \mu \mathrm{m}$ \\
\hline$L_{2 x}$ & $400 \mu \mathrm{m}$ & $t$ & $20 \mu \mathrm{m}$ \\
\hline$L_{C}$ & $1600 \mu \mathrm{m}$ & $w$ & $10 \mu \mathrm{m}$ \\
\hline
\end{tabular}

The equivalent stiffness of the suspension systems and the resonant frequencies are given in Table II.

TABle II. The PARAmeters CAlCUlated Using EQ. 1-9

\begin{tabular}{|c|c|c|c|c|c|}
\hline Parater & Value & Parater & Value & Paramer & Value \\
\hline$K_{1 x}$ & $\begin{array}{c}13.34 \\
\mathrm{~N} / \mathrm{m}\end{array}$ & $K_{1 y}$ & $12.06 \mathrm{~N} / \mathrm{m}$ & $m_{1}+m_{3}$ & $\begin{array}{c}0.7446 \mathrm{e}-6 \\
\mathrm{~kg}\end{array}$ \\
\hline$K_{2 x}$ & $\begin{array}{c}24.8 \\
\mathrm{~N} / \mathrm{m}\end{array}$ & $K_{2 y}$ & $17.17 \mathrm{~N} / \mathrm{m}$ & $w_{x}$ & $\begin{array}{c}1996.92 \\
\mathrm{~Hz}\end{array}$ \\
\hline$k c$ & 3.36 & $m_{1}+m_{2}$ & $\begin{array}{c}1.0509 \mathrm{e}-6 \\
\mathrm{~kg}\end{array}$ & $w_{y}$ & $\begin{array}{c}1994.36 \\
\mathrm{~Hz}\end{array}$ \\
\hline
\end{tabular}

\section{B. Numerical Analysis for Determination of Natural Frequency}

In this section, resonant frequency in the drive and sense mode is calculated using COMSOL modal analysis.

Fig. 3 and Fig. 4 show the drive mode shape at the resonant frequency of $1998.3 \mathrm{~Hz}$ and sense mode shape of 2001.16 $\mathrm{Hz}$.

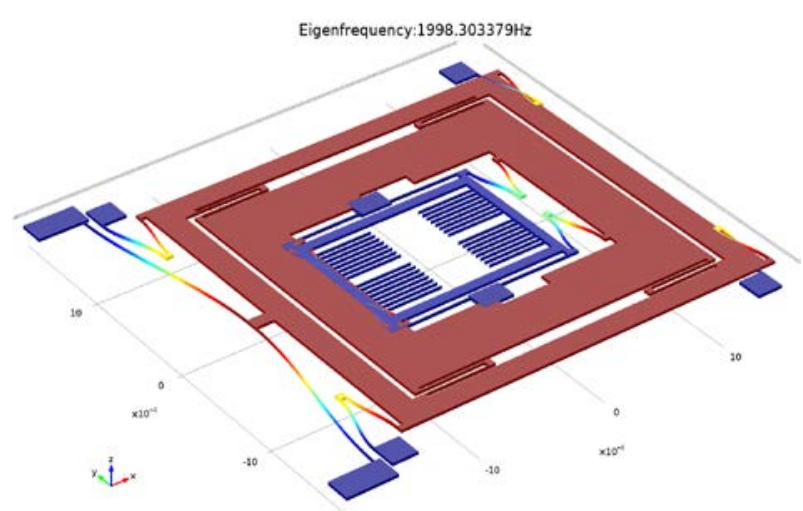

Fig. 3. Modal analysis (夰ive mode shape at $1998 \mathrm{~Hz}$ ).

The results in Section III.A and III.B are compared in Table. III and show good agreement between them.
TABLE III. THE RESONANT FREQUENCY OBTAINED USING BOTH ANALYTICAL AND NUMERICAL (MENTIONED AS COMSOL) METHODS.

\begin{tabular}{|c|c|c|c|}
\hline & Analytical & Comsol & Mismatch \\
\hline Drive mode & $1997 \mathrm{~Hz}$ & $1998 \mathrm{~Hz}$ & 0.05 \\
\hline Sense mode & $1994 \mathrm{~Hz}$ & $2001 \mathrm{~Hz}$ & 0.35 \\
\hline
\end{tabular}

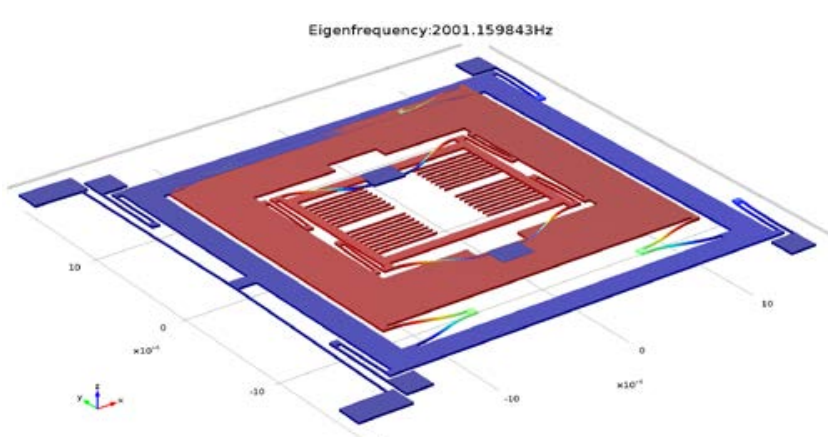

Fig. 4. Modal analysis (Sense mode shape at $2001 \mathrm{~Hz}$ ).

\section{HEAT TRANSFER ANALYSIS}

By applying a voltage to an electrothermal microactuator, its temperature goes up. However, heat transfer effect doesn't allow the temperature to be increased as that much. In order to analysis the heat transfer effect in a v-shaped electrothermal actuator, a model is used according to Fig. 5 [14].

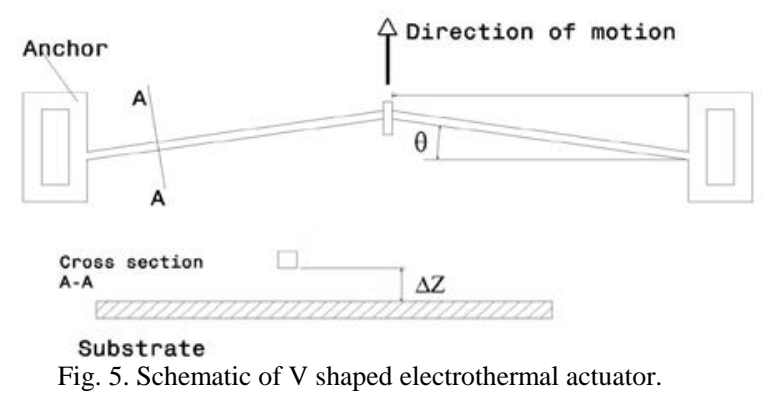

While the length of the microactuator is much larger than its width and thickness, it is modeled as a one-dimensional heat transfer problem. Since then, the temperature in each cross-section is assumed to be constant. The gap between the hot arm and the substrate ( $\Delta Z$ ) is very small and the gradients of temperature in this gap are very large. So the conduction heat transfer via the atmosphere around the hot arm becomes highly important. In this case, the conduction heat transfer coefficient, $\mathrm{U}$, to the substrate can be calculated by Eq. 10 [14].

$$
U=K_{\text {air }} / \Delta Z
$$

In Eq. $10 K_{a i r}$ is air thermal conductivity which is equal to $0.03 W \cdot m^{-1} \cdot K^{-1}$ in the room temperature [16]. If $\Delta Z=5 \mu m$, the value for $U$ will be $6000 \mathrm{~W} \cdot \mathrm{m}^{-2} \cdot \mathrm{K}^{-1}$ which shows conduction heat transfer plays an important role in this kind of actuator.

\section{A. The Performance of Drive Mode of Microgyroscope}

Since this microgyroscope was designed based on application of electrothermal actuator in the drive mode, the performance of the actuator is investigated in this section.

The function of this actuator is based on increasing in length of the arms caused by increasing in temperature when 
the difference in voltage across the anchors is appeared. This expansion in arms leads to a displacement in drive direction.

Under different applied voltages, the variations in temperature and therefore the displacement induced in the arms of the actuators are different. In Fig. 6 the displacement in drive direction is shown vs. different values of voltage.

The function of a MEMS vibratory gyroscope in drive mode is based on exciting it by a constant frequency. So the applied voltage should be able to induce sinusoidal force which causes sinusoidal vibration by the arms when their temperature differs.

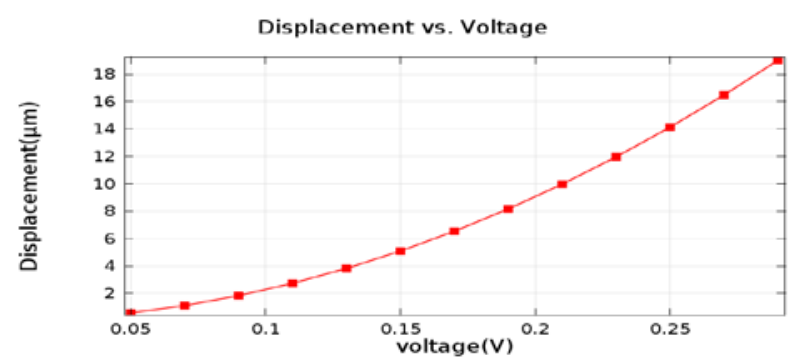

Fig. 6. Displacement response to voltage change.

Therefore, an alternative voltage should be used. We should excite the gyroscope by the frequency close to resonant frequency of the drive mode to have maximum displacement.

To determine the maximum drive displacement, first, the equivalent force induced by electrical excitation should be calculated according the COMSOL standards.

To determine the equivalent force induced by electrical excitation, we plotted the displacement vs. different DC voltages. In fact, we modeled the $\mathrm{AC}$ voltages by a DC voltage which varies over time according to Eq. 11. The boundary conditions for each step in the simulation are derived from the values of the variables in the previous step.

$$
V_{A C}=V_{D C} \times \sin (w t)
$$

Considering $V_{D C}=0.18$ and $w=1000 \mathrm{~Hz}$ in Eq. 11, the induced displacement in the gyroscope is plotted in the time interval of [0s, 0.04s] using the time step of $0.00005 \mathrm{~s}$. As it is shown in Fig.7, after short time the displacement amplitude and frequency become constant. In fact, after a short time, we have sinusoidal vibration which shows the force is also sinusoidal.

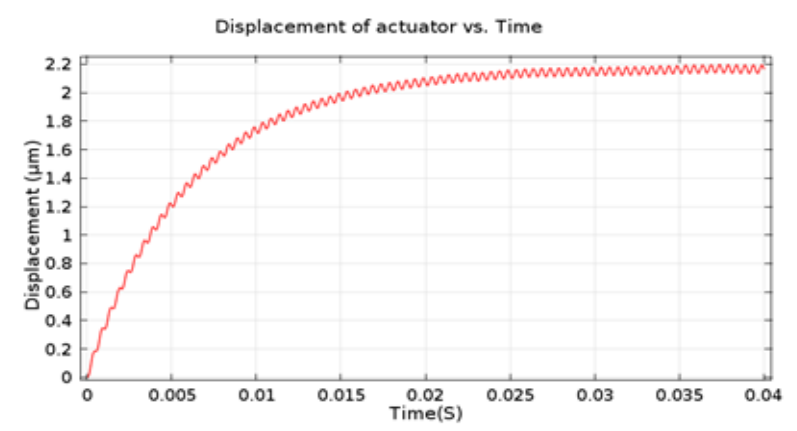

Fig. 7. The plot of the displacement of the microgyroscope.

Fig. 8 shows the induced displacement in a short time interval after being stable.

Since the function of electrothermal actuator is independent of the polarity of the voltage being applied, each period of AC voltage causes two periods of displacement [14]. Then, in our case, the frequency of displacement is $2000 \mathrm{~Hz}$.

The induced displacement has an offset value of $2.15 \mu \mathrm{m}$ and peak-to-peak displacement of $0.06 \mu \mathrm{m}$ and the frequency of $2000 \mathrm{~Hz}$. The force which is equivalent to the alternative displacement is calculated using force-displacement plot and is shown in Fig. 9. The equivalent force that is induced in the arms when a 0.18-volt excitation with the frequency of 1000 $\mathrm{Hz}$ is an alternative force with the amplitude of 4.7e-6 $\mathrm{N}$ and the frequency of $2000 \mathrm{~Hz}$.

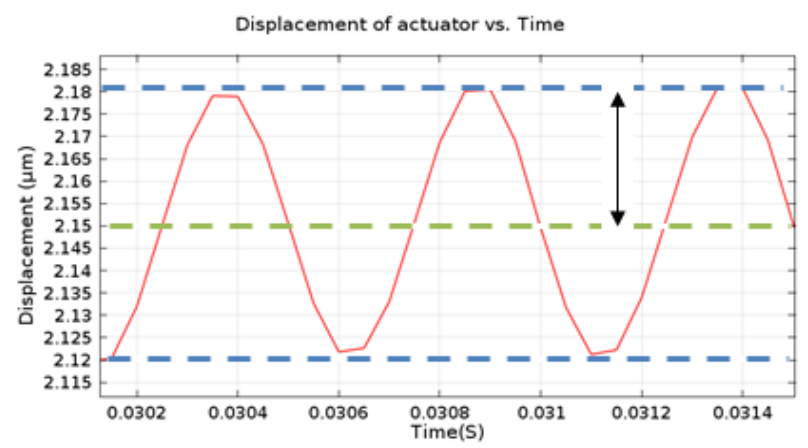

Fig. 8 . The higher temporal resolution plot of the displacement after being stable.

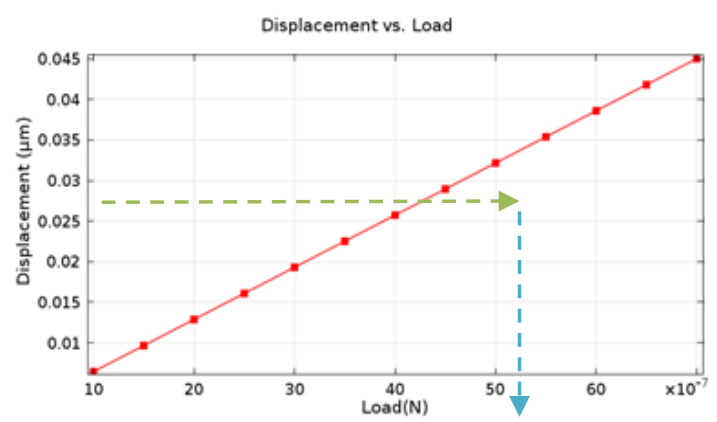

Fig. 9. The plot shows the displacement vs. the applied load.

\section{DAMPING}

The total damping In a micromachined vibratory gyroscope consists of many dissipation mechanisms [6]. Since the damping caused by the surrounding gas is noticeable among the other damping mechanisms, we only considered the damping of gas. When the vibratory structure of a microgyroscope is surrounded by a gas, the viscous effect of the gas between the proof mass and the stationary surfaces provides the primary damping mechanism in the gyroscope dynamical systems.

Regarding the designed structure and direction of oscillation in our proposed microgyroscope, only slide film damping should be considered which can be calculated using Eq.12.

$$
C_{\text {slide }}=\mu \frac{A}{d}
$$

In Eq.12 $\mu$ is the viscosity coefficient, $A$ is the area and $d$ is the gap.

\section{FREQUENCY RESPONSE IN DRIVE MODE}

We modeled the microgyroscope using 
mass-spring-damper system as shown in Fig. 10 and the equation governing in the drive mode is described in Eq.13.

$$
m_{d} \ddot{x}+c_{d} \dot{x}+k_{d} x=F_{0} \sin w t
$$

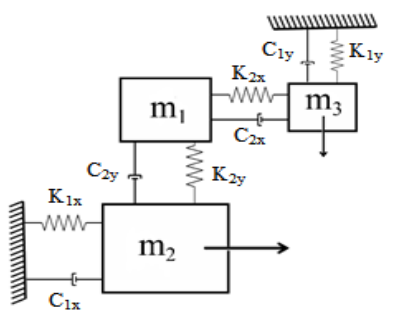

Fig. 10. Lumped mass-spring-damper model.

Fig. 11 shows the frequency response of the drive mode when the force, which is induced by applying an alternative voltage whose amplitude is $0.18 \mathrm{~V}$ and frequency is $1000 \mathrm{~Hz}$, is applied to it. And the damping factor is calculated using Eq. 12.

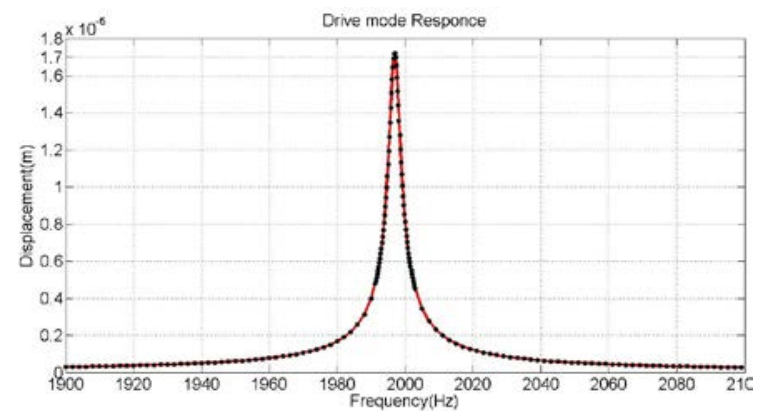

Fig. 11. Frequency response of the drive mode.

In addition, the frequency response is calculated using comsol and is shown in Fig. 12. Both numerical and analytical results are in good agreement.

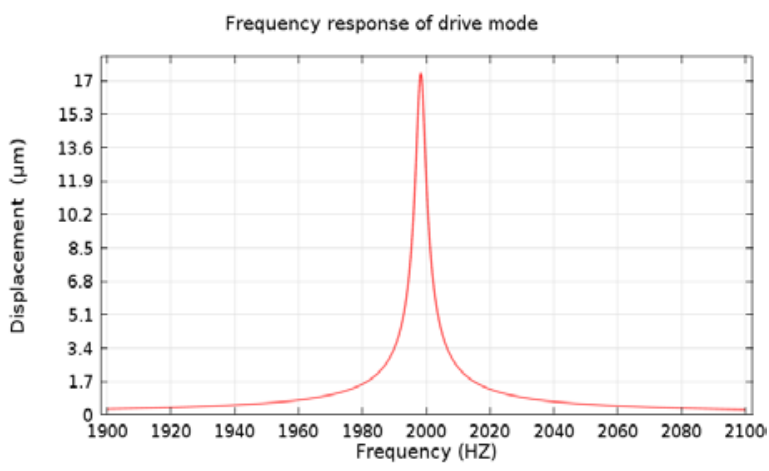

Fig. 12. Frequency response of the drive mode.

As it shown in Fig. 11 and Fig. 12, if the proposed microgyroscope is excited around the resonant frequency of the drive mode, the maximum displacement will be obtained by which the drive mode amplitude will be appropriate.

\section{CONCLUSION}

This paper presented an electrothermally actuated MEMS vibratory gyroscope. To increase the performance, the structure of the gyroscope is designed such that, the resonant frequency of the drive mode and the sense mode are close to each other. The function of the proposed gyroscope is simulated with a sinusoidal voltage input whose amplitude and frequency are $0.18 \mathrm{~V}$ and $1000 \mathrm{~Hz}$ respectively. The results show that the microgyroscope generates a displacement with the amplitude of 12 micron and frequency of $2000 \mathrm{~Hz}$. This makes it suitable to be used as a microgyroscope actuator for many applications.

\section{REFERENCES}

[1] X. Liu, Z. Yang, X. Chi, J. Cui, H. Ding, and Z. Guo et al., "An X-axis micromachined gyroscope with doubly decoupled oscillation modes," in Proc. IEEE 21st International Conference on Micro Electro Mechanical Systems, 2008, pp. 860-863.

[2] N. Yazdi, F. Ayazi, and K. Najafi, "Micromachined inertial sensors," Proceedings of the IEEE, vol. 86, 1998, pp. 1640-1659.

[3] J. Georgy, A. Noureldin, M. J. Korenberg, and M. M. Bayoumi, "Modeling the stochastic drift of a MEMS-based gyroscope in gyro/odometer/GPS integrated navigation," Intelligent Transportation Systems, IEEE Transactions on, vol. 11, pp. 856-872, 2010.

[4] R. Neul, U.-M. Gomez, K. Kehr, W. Bauer, J. Classen, and C. Doring et al., "Micromachined angular rate sensors for automotive applications," Sensors Journal, IEEE, vol. 7, pp. 302-309, 2007.

[5] K. Riaz, S. A. Bazaz, M. M. Saleem, and R. I. Shakoor, "Design, damping estimation and experimental characterization of decoupled 3-DoF robust MEMS gyroscope," Sensors and Actuators A: Physical, vol. 172, pp. 523-532, 2011.

[6] C. Acar and A. Shkel, "MEMS vibratory gyroscopes: structural approaches to improve robustness," Springer Science \& Business Media, 2008.

[7] A. Sharaf, S. Sedky, and S. Habib, "Complete analysis of a novel fully symmetric decoupled micromachined gyroscope," in Proc. 2006 International Conference on MEMS, NANO and Smart Systems, 2006, pp. 35-40.

[8] W. Geiger, J. Merz, T. Fischer, B. Folkmer, H. Sandmaier, and W. Lang, "The silicon angular rate sensor system MARS-RR," in Proc. the 10th International Conference on Solid-State Sensors and Actuators, Transducers, 1999, pp. 1578-1581.

[9] W. Geiger, W. Butt, A. Gaisser, J. Frech, M. Braxmaier, and T. Link et al., "Decoupled microgyros and the design principle DAVED," Sensors and Actuators A: Physical, vol. 95, pp. 239-249, 2002.

[10] A. Sharaf, S. Sedky, M. Serry, A. Elshurafa, M. Ashour, and S. Habib, "A fully symmetric and completely decoupled MEMS-SOI gyroscope," Sensors \& Transducers, vol. 127, pp.1726-5479, 2011.

[11] S. E. Alper and T. Akin, "A single-crystal silicon symmetrical and decoupled MEMS gyroscope on an insulating substrate," Journal of Microelectromechanical Systems, vol. 14, pp. 707-717, 2005.

[12] S. E. Alper, K. M. Silay and T. Akin, "A low-cost rate-grade nickel microgyroscope," Sensors and Actuators A: Physical, vol. 132, pp. 171-181, 2006

[13] R. I. Shakoor, S. A. Bazaz, M. Burnie, Y. Lai, and M. Hasan, "Electrothermally actuated resonant rate gyroscope fabricated using the MetalMUMPs," Microelectronics Journal, vol. 42, pp. 585-593, 2011.

[14] R. Hickey, D. Sameoto, T. Hubbard, and M. Kujath, "Time and frequency response of two-arm micromachined thermal actuators," Journal of Micromechanics and Microengineering, vol. 13, p. 40, 2003.

[15] J. J. Luo, J. H. He, A. Flewitt, D. Moore, S. M. Spearing, and N. A. Fleck et al., "Development of all metal electrothermal actuator and its applications," Journal of Micro/Nanolithography, MEMS, and MOEMS, vol. 4, pp. 023012-023012-10, 2005.

[16] F. P. Incropera, Fundamentals of Heat and Mass Transfer, John Wiley \& Sons, 2011.

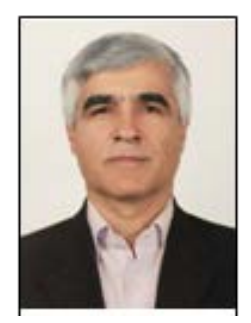

Mohsen Hamedi is a professor of manufacturing in School of Mechanical Engineering, University of Tehran, Iran where he teaches graduate and undergraduate courses. He obtained his $\mathrm{PhD}$ in 1995 from UNB, Canada and M.Sc. and B.Sc. from University of Tehran respectively. Dr. Hamedi has supervised more than $60 \mathrm{PhD}$ and M.Sc. dissertations. He has published more than 120 papers in the refereed international journals and conferences. His research area of interests are micro-sensor and micro-actuator design and fabrication, mechanica energy harvesting and manufacturing process optimization. 